\title{
Partial tetrasomy 12pter-12p12.3 in a girl with Pallister-Killian syndrome: extraordinary finding of an analphoid, inverted duplicated marker
}

\author{
Andreas Dufke*,1, Claudia Walczak ${ }^{1}$, Thomas Liehr ${ }^{2}$, Heike Starke ${ }^{2}$, Vladimir Trifonov ${ }^{2,3}$, \\ Nikolai Rubtsov ${ }^{2,3}$, Martin Schöning ${ }^{4}$, Herbert Enders ${ }^{1}$ and Thomas Eggermann ${ }^{5}$ \\ ${ }^{1}$ Abteilung Medizinische Genetik, Klinikum der Eberhard-Karls-Universität Tübingen, Germany; ${ }^{2}$ Institut für \\ Humangenetik und Anthropologie, Klinikum der Friedrich-Schiller-Universität Jena, Germany; ${ }^{3}$ Institute of \\ Cytology and Genetics, Novosibirsk, Russia; ${ }^{4}$ Abteilung für Sozialpädiatrie und Entwicklungsneurologie, \\ Kinderklinik der Eberhard-Karls-Universität Tübingen, Germany; ${ }^{5}$ Institut für Humangenetik, Universitätsklinikum \\ der RWTH Aachen, Germany
}

Cytogenetic analysis in a girl with multiple congenital anomalies indicating Pallister-Killian syndrome (PKS) showed a supernumerary marker chromosome in 1/76 lymphocytes and 34/75 fibroblast metaphases. GTGbanding pattern was consistent with the chromosomal region 12pter-12q11. While fluorescence-in-situ hybridisation (FISH) with a whole chromosome 12 painting probe confirmed the origin of the marker, a chromosome 12 specific $\alpha$-satellite probe did not hybridise to it. FISH analysis with a specific subtelomeric probe $12 p$ showed hybridisation to both ends of the marker chromosome. High-resolution multicolour-banding $(\mathrm{MCB})$ studies revealed the marker to be $\mathrm{a} \operatorname{der}(12)(\mathrm{pter} \rightarrow \mathrm{p} 12.3: \mathrm{:p12.3 \rightarrow pter).} \mathrm{Summarising} \mathrm{the} \mathrm{FISH}$ information, we defined the marker as an inverted duplication of 12pter-12p12.3 leading to partial tetrasomy of chromosome $12 \mathrm{p}$. In skin fibroblasts, cultured at the patient's age of 1 year and 9 years, the marker chromosome was found in similar frequencies, even after several culture passages. Therefore, we consider the marker to have a functional centromere although it lacks detectable centromeric $\alpha$-satellite sequences. To the best of our knowledge, this is the first proven analphoid marker of chromosome 12. Molecular genetic studies indicated that this marker is of paternal origin. The finding of partial tetrasomy 12pter-12p12.3 in our PKS patient allows to narrow down the critical region for PKS. European Journal of Human Genetics (2001) 9, $572-576$.

Keywords: Pallister-Killian syndrome (PKS); partial tetrasomy 12p; analphoid marker chromosome; PKS critical region; multicolour banding $(\mathrm{MCB})$

\section{Introduction}

PKS, a rare disorder first described by Pallister et al ${ }^{1}$ and later by Teschler-Nicola and Killian, ${ }^{2}$ is characterised by tetrasomy of the complete short arm of chromosome 12. Phenotypic expression in this disorder varies from multiple anomalies resulting in perinatal death to the more frequent situation

*Correspondence: A Dufke, Abteilung Medizinische Genetik, Institut für Anthropologie und Humangenetik, Wilhelmstr. 27, D-72074 Tübingen, Germany. Tel: +49 7071 2976408; Fax: +49 7071 295228;

E-mail: andreas.dufke@uni-tuebingen.de

Received 23 February 2001; revised 23 April 2001; accepted 2 May 2001 with sparse hair of temporal alopecia, 'coarse' face, pigmentary dysplasia, short and broad hands, severe mental retardation and seizures. ${ }^{3}$ The characteristic additional isochromosome $12 \mathrm{p}[\mathrm{i}(12 \mathrm{p})]$ is usually restricted to fibroblasts; it is rarely found in metaphases of peripheral blood. In the more than 100 patients that have been described so far, the supernumerary $\mathrm{i}(12 \mathrm{p}) \mathrm{s}$ contained the whole short arm of chromosome 12 . There is only a single report with an apparently analphoid isochromosome $12 \mathrm{p}$ in the literature. ${ }^{4}$ A specific region, which might be responsible for the PKS phenotype when present in tetrasomic state, has not yet been described. 
Here we report a girl presenting clinical signs of PKS. Cytogenetic and molecular analyses revealed an unusual analphoid marker chromosome of paternal origin, which could be characterised as an inverted duplication 12pter12p12.3. The resulting distal tetrasomy $12 \mathrm{p}$ narrows down the PKS critical region to 12 pter-12p12.3.

\section{Patient}

The girl was born in 1991 as the third child of healthy nonconsanguineous Italian parents. She has two healthy older brothers and a younger healthy sister. After an uneventful pregnancy, delivery was spontaneous at term (birth weight $3.300 \mathrm{~g}$ (50th centile), length $49 \mathrm{~cm}$ (25th centile), head circumference (OFC) $33 \mathrm{~cm}$ (10th centile), Apgar scores 10/ 10/10).

At the age of 1 month she was referred to a children's hospital because of apparent dysmorphic signs and heart murmur. She was hypotonic. Weight, length and OFC were between the 3rd and 7th centile. Anomalies included small slightly low set ears, hypertelorism, narrow palpebral fissures, simian crease on the right hand, asymmetric folds of the legs and a sacral dimple. Echocardiography revealed an apparently narrow pulmonary artery, a slight supraventricular stenosis and a small posterio-apical situated atrial septal defect. Ultrasound of the hips showed a dislocation on the left side.

We first saw the girl at the age of 1 year. At this time her weight was $11 \mathrm{~kg}$ (97th centile), length $71 \mathrm{~cm}$ (10th centile) and OFC $43.5 \mathrm{~cm}$ (3rd centile). Craniofacial dysmorphisms now included bitemporal sparsity of hair, narrow prominent forehead, small ears, ptosis, hypertelorism, broad flat nasal bridge, small nose, long philtrum, thin upper lip, drooping lower lip and high arched palate. She had broad hands with terminal hypoplasia of fingers. Developmental retardation was obvious. These clinical findings strongly suggested the diagnosis of PKS (Figure 1). At the age of 1 year, 7 months, severe global retardation, generalised muscular hypotonia, microcephaly and obesity were noted. Seizures have been present since the age of 3 years. At the age of $6 \frac{1}{2} 2$ years, body measurements were: height $115 \mathrm{~cm}$ (10th centile), weight $30.1 \mathrm{~kg}$ (97th centile) and OFC $47.9 \mathrm{~cm}$ (<3rd centile). There was no speech. No pigmentary dysplasia could be observed.

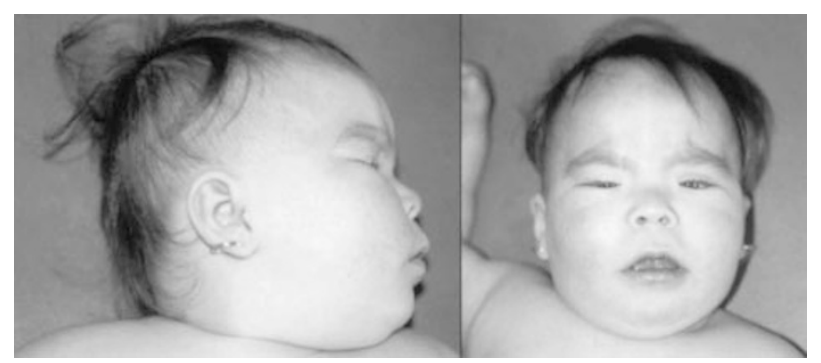

Figure 1 Proposita at the age of $1 \frac{1}{2}$ years.
Analysis of lactate-dehydrogenase (LDH) activity in blood was within the normal range. In cultured fibroblasts, LDH-B expression was not increased in relation to LDH-A.

\section{Materials and methods Cytogenetics}

Metaphase spreads were prepared from skin fibroblast cultures of the patient and from peripheral blood lymphocytes of the patient and her parents following standard protocols. GTG-, C- and NOR-banding were performed according to standard procedures.

\section{Molecular cytogenetics}

FISH studies on metaphase spreads of cultured fibroblasts using whole chromosome 12 painting probe (WCP12), 12p telomeric probe (TelVysion $12 \mathrm{p}$, STS sAVH27) and $\alpha$-satellite centromeric probe (CEP12), were carried out according to the manufacturer's instructions (Vysis Inc., Downers Grove, IL, USA).

High resolution multicolour banding (MCB) technique using a chromosome 12-specific DNA probe cocktail was performed according to Chudoba et al. ${ }^{5}$

\section{Molecular genetics}

To determine the parental origin and the mechanism of marker formation, we studied the derivative $12 \mathrm{p}$ by short tandem repeat (STR) typing. DNA of the patient and her parents was extracted according to standard procedures from fibroblasts and from blood, respectively. The typed STR markers are listed in Table 1 . Primer sequences and amplification conditions were obtained from the Genome Database. ${ }^{6}$ After denaturing sequencing gel electrophoresis the alleles were visualised by silver-staining.

\section{Results}

Cytogenetic analysis of peripheral blood lymphocytes was performed in the newborn period. Analysis of 75 metaphase spreads revealed a normal female karyotype with a variant short arm of one chromosome 15 $(46, \mathrm{XX}, 15 \mathrm{p}+)$. This structural variant was ascertained by NOR-, C-, and Q-banding. In one metaphase a supernumerary marker chromosome was found which was consistent with that identified later in fibroblasts. Parental karyotypes were normal except for a paternal variant chromosome $15 \mathrm{p}+$.

At the age of 1 year metaphase spreads from skin fibroblasts were analysed. In 50\% of GTG-banded metaphases a supernumerary marker chromosome was found \{karyotype: 47,XX,15p+,+mar[13]/46,XX,15p+[13]\}. The marker showed a primary constriction and resembled in size and shape an acrocentric chromosome of the G-group (Figure 2A). A whole chromosome 12 painting probe stained the marker chromosome completely. 
Table 1 Results of STR typing studies in the presented case with the supernumerary marker chromosome 12pter$12 \mathrm{p} 12.3$

\begin{tabular}{llllll}
\hline STR & Localisation & Father & Mother & Patient & Informativity \\
\hline 12pter & & & & & \\
D12S94 & $12 \mathrm{p} 13$ & $1-2$ & $1-1$ & $1-2^{*}$ & Paternal, R \\
D12S1685 & 12p13 & 1 & 1 & 1 & - \\
D12S99 & $12 \mathrm{p} 13$ & 1 & 1 & 1 & - \\
D12S356 & $12 \mathrm{p} 13$ & $1-2$ & 1 & $1-2^{*}$ & Paternal, R \\
D12S336 & $12 \mathrm{p} 12-\mathrm{p} 13$ & 1 & 1 & 1 & - \\
D12S77 & $12 \mathrm{p} 12$ & $1-2$ & $1-3$ & 1 &,$- \mathrm{R}$ \\
D12S1697 & $12 \mathrm{p} 11-12$ & $1-3$ & $2-4$ & $2-3^{*}$ & Paternal, R \\
Cen & & & & & \\
D12S87 & $12 \mathrm{p} 13-\mathrm{q} 24.1$ & $1-2$ & $1-2$ & 1 & - \\
D12S83 & $12 \mathrm{q}$ & $2-4$ & $1-3$ & $3-4$ & Biparental \\
12qu & & & & &
\end{tabular}

12 qter

All informative markers in $12 p$ showed reduction $(R)$ of paternal heterozygosity to homozygosity in the patient. The genetic order of markers was obtained from the Généthon human linkage map. ${ }^{18}$ (*Band intensities indicate the presence of more than one copy of the allele).

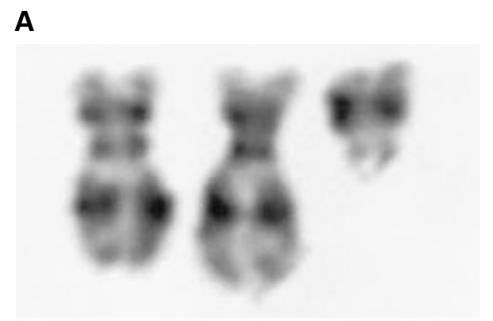

B

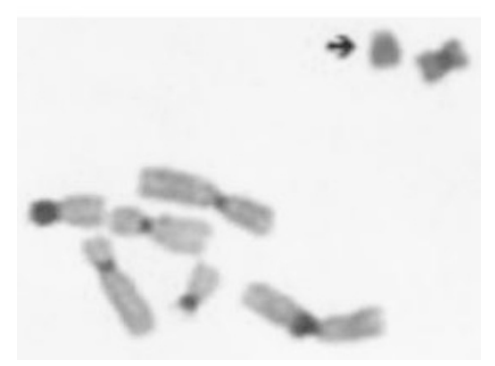

Figure 2 Results of the cytogenetic analysis from cultured fibroblasts. (A) GTG-banded chromosome 12 pair and the derivative chromosome 12. (B) C-banding does not stain the centromeric region of the marker chromosome (arrow).

Fibroblast cell lines of the now 9 year-old patient were established. GTG-banding showed an almost identical mosaic state compared to the initial investigation \{karyotype: $47, \mathrm{XX}, 15 \mathrm{p}+,+\operatorname{mar}[28] / 46, \mathrm{XX}, 15 \mathrm{p}+[21]\}$. C-banding failed to show heterochromatin of the centromeric region on the marker chromosome (Figure 2B).

After hybridisation with the WCP12, TelVysion 12p and CEP12 probes, the expected signal distributions were seen on the normal chromosomes 12 . The marker chromosome was stained completely with the WCP12 probe. No signal for the centromeric region could be detected with the CEP12 probe. The TelVysion $12 p$ probe hybridised to both ends of the marker chromosome, indicating tetrasomy for the subtelomeric region of $12 p$ (Figure 3 ). MCB finally characterised the marker chromosome as $\operatorname{der}(12)($ pter $\rightarrow$ p12.3::p12.3 $\rightarrow$ pter $)$ (Figure 4).

These studies have been performed after several passages of fibroblast cultures and virtually no differences in the proportion of cells containing the marker chromosome could be observed.

The STR typing results indicated a paternal origin of the marker chromosome (Table 1). In the case of STRs D12S94, D12S356 and D12S1697 the bands corresponding to the paternally inherited alleles showed stronger intensities than the maternal one. Additionally, the STRs D12S94, D12S356, D12S77 and D12S1697 showed reduction of paternal heterozygosity to homozygosity in the patient. A maintenance of paternal heterozygosity was not observed. Biparental origin of the normal chromosome 12 homologues was demonstrated by long arm STR typing.

\section{Discussion}

PKS associated with tetrasomy of the complete short arm of chromosome 12 has been described in more than 100 patients. The distinct phenotype of PKS enables a diagnosis based solely on clinical manifestations in the majority of cases. ${ }^{3,7}$ In our patient, the clinical findings were consistent with PKS. Nevertheless, cytogenetic analysis did not reveal the typical finding of supernumerary $\mathrm{i}(12 \mathrm{p})$ in fibroblasts. By applying molecular-cytogenetic methods, we demonstrated the marker chromosome to be an inverted duplication of the region 12pter-12p12.3. We therefore propose a smallest critical region of tetrasomy 12 pter-12p12.3 to result in the clinical phenotype of PKS.

Before the introduction of molecular-cytogenetic methods unequivocal characterisation of marker chromosomes only based on their banding patterns could be challenging. Therefore, in the past, identification of an extra chromosome as derivative chromosome $12 \mathrm{p}$ had been confirmed by dosage effect of the LDH-B gene which is located within $12 \mathrm{p} 12.1 \rightarrow \mathrm{p} 12.2 .^{8,9}$ Our case illustrates that even the combination of chromosome banding methods and LDH-B measurements might not have identified all PKS patients. This is of special interest since the clinical phenotype of PKS is in some instances difficult to recognise, ie in prenatal ultrasound examination and in prematurely born neonates. The finding of an unusual marker chromosome should not exclude PKS as possible diagnosis until further molecularcytogenetic analyses are carried out.

FISH with a chromosome 12 specific $\alpha$-satellite probe did not show hybridisation to the marker chromosome in our case, indicating that it lacked detectable $\alpha$-satellite sequences. This was also confirmed by C-banding. The marker chromo- 


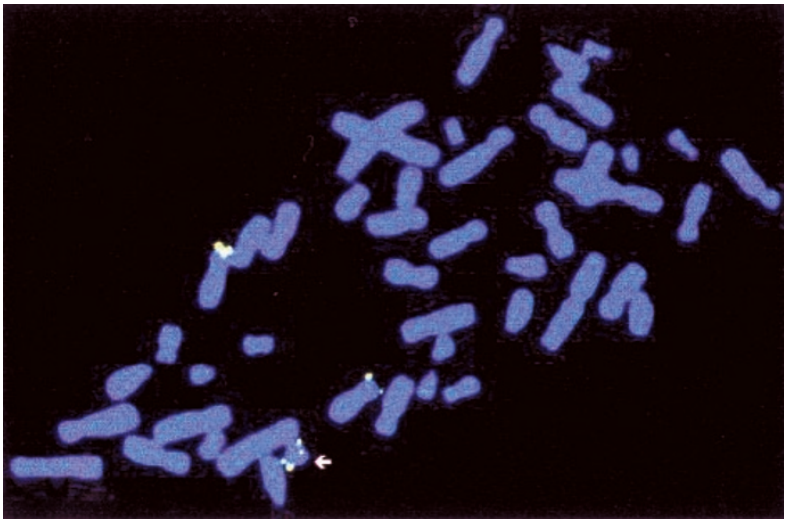

Figure 3 FISH-analysis with the TelVysion 12p probe (Vysis Inc.) on a metaphase spread of cultured fibroblasts giving signals on both ends of the derivative chromosome 12 (arrow). some was found in cultured fibroblasts from skin biopsies, carried out at the age of 1 year and 9 years in 50 and $57 \%$ of cells, respectively. Even after several passages of the fibroblast cultures, virtually no loss of the extra chromosome could be observed. We therefore consider the analphoid marker to be mitotically stable at least in slowly proliferating tissue. Analphoid supernumerary marker chromosomes have been reported in more than 25 patients. ${ }^{10}$ They have been reported for several autosomes and the Y chromosome, and have occurred predominantly as isochromosomes or inverted duplications and less frequently as ring chromosomes or apparently linear fragments (for review see ${ }^{11}$ ). Like in the presented case, these markers have always lacked detectable C-band positive centromeric heterochromatin and $\alpha$-satellite DNA. Furthermore, originally acentric chromosome fragments (mostly inverted duplicated short arm chromosomes)

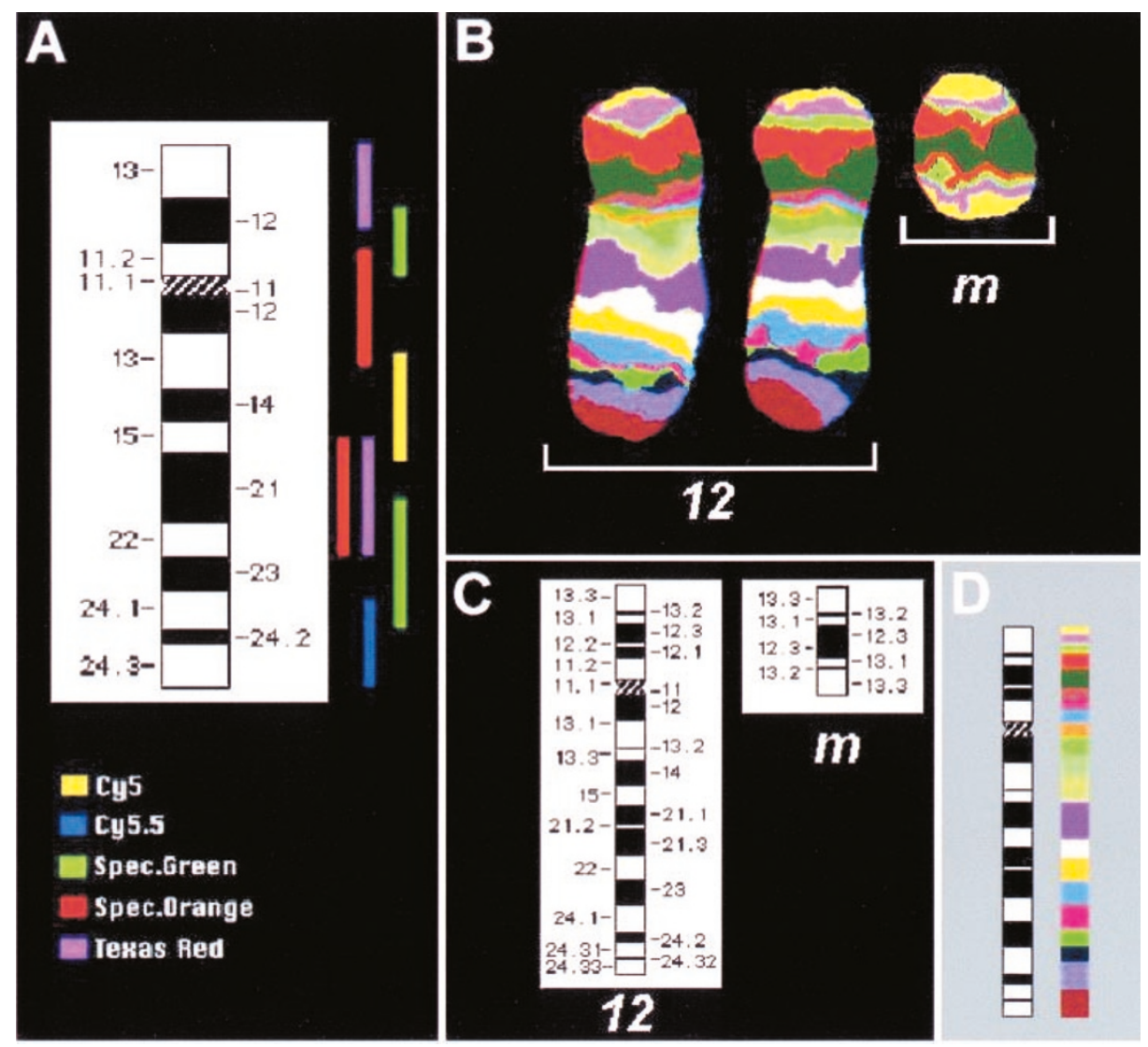

Figure 4 Result of high resolution multicolour banding (MCB) after application of a chromosome 12 specific probe cocktail. The localisation of the chromosome 12 region specific partial chromosome paints and the used fluorochromes are given in (A). Images were captured on a Zeiss Axioplan microscope equipped with the ISIS digital FISH imaging system (MetaSystems, Altlussheim, Germany) using a XC77 CCD camera with on-chip integration (Sony). The resulting pseudocolour MCB patterns for the two normal chromosomes 12 and the derivative chromosome $12(\mathrm{~m})$ of the presented PKS patient are shown in (B). The MCB-pattern has been aligned to the GTGbanding pattern and thus allows the location of the breakpoint; the dark green band in which the break and fusion in the marker took place corresponds to the band 12p12.3. The centromere of the normal chromosomes 12 is located in the orange band. Ideograms based on ISCN 1995, 550 bands per haploid karyotype level, for the normal and for the derivative chromosome 12 are depicted in (C). In (D) the alignment of GTG and MCB banding is shown. 
have been reported, which must have gained stable centromere function at chromatin devoid of $\alpha$-satellite DNA sequences. Due to their mitotic stability, it was proposed that these analphoid markers contained neocentromeres, a region of chromatin that may act as a functional centromere. ${ }^{12}$

The mechanism of formation of the marker chromosome in our patient appears to be similar to that of two cases published by Depinet et al. ${ }^{13}$ The analphoid marker $12 \mathrm{p}$ is of paternal origin; reduction of paternal heterozygosity to homozygosity in all analysed STRs indicates a postzygotic mitotic error. Summarising the so far published data on the mechanism of formation of analphoid, inverted duplicated markers, postzygotic mitosis as cell stage of origin has been described three times, while maternal or paternal meiotic errors could each be delineated only once. ${ }^{13,14}$ Analphoid inverted duplicated markers may be generated by a single ' $U$ type' exchange event involving chromosome breakage and subsequent reunion between the sister chromatids. A ' $U$ type' exchange can also be generated by a simple abnormal event in DNA replication, as suggested by Sjösted et al. ${ }^{15}$ Conversely, isochromosomes of the complete chromosome $12 \mathrm{p}$ as well as other short arm isochromosomes mainly originate from maternal meiosis II errors followed by rearrangements leading to duplication of the short and loss of the long arm. ${ }^{16,17}$

In conclusion, the case presented here allows to narrow down the PKS critical region to 12pter-12p12.3. Furthermore, it is the first case with proven analphoid marker of chromosome 12p.

\section{Acknowledgements}

We thank Prof Dr J Kömpf for LDH diagnosis.

\section{References}

1 Pallister PD, Meisner LF, Elejalde BR et al: The Pallister mosaic syndrome. BDOAS 1977; 13: $103-110$.

2 Teschler-Nicola M, Killian W: Case Report 72: Mental retardation, unusual facial appearance, abnormal hair. Synd Ident 1981; 7: $6-7$.

3 Schaefer GB, Jochar A, Muneer R, Sanger WG: Clinical variability of tetrasomy 12p. Clin Genet 1997; 51: 102-108.
4 Speleman F, Leroy JG, Van Roy $\mathrm{N}$ et al: Pallister-Killian syndrome: Characterization of the Isochromosome $12 p$ by fluorescent in situ hybridization. Am J Med Genet 1991; 41: $381-387$.

5 Chudoba I, Plesch A, Lörch T, Lemke J, Claussen U, Senger G: High resolution multicolor-banding: a new technique for refined FISH analysis of human chromosomes. Cytogenet Cell Genet 1999; 84: 156-160.

6 Genome Database: http://www.gdb.org

7 Reynolds JF, Daniel A, Kelly TE et al: Isochromosome 12p mosaicism (Pallister mosaic aneuploidy or Pallister-Killian syndrome): Report of 11 cases. Am J Med Genet 1987; 27: 257 274.

8 Steinbach P, Rehder H: Tetrasomy for the short arm of chromosome 12 with accessory isochromosome $(+\mathrm{i}(12 \mathrm{p}))$ and a marked LDH-B gene dosage effect. Clin Genet 1987; 32: 1-4.

9 Rethoré MO, Kaplan JC, Junien C et al: Augmentation de l'activité de la LDH-B chez un garçon trisomique 12p par malségregation d'une translocation maternelle t(12:14)(q12;p11). Ann Génét 1975; 18: 81-87.

10 Rivera H, Vasquez AI, Garcia-Cruz D, Crolla JA: Neocentromere at $13 \mathrm{q} 32$ in one of two stable markers derived from a $13 \mathrm{q} 21$ break. Am J Med Genet 1999; 85: $385-388$.

11 Choo KHA: Chromatin dynamics '97: Centromere DNA dynamics: Latent centromeres and neocentromere formation. Am J Hum Genet 1997; 61: 1225 - 1233.

$12 \mathrm{du}$ Sart D, Cancilla MR, Earle E et al: A functional neocentromere formed through activation of a latent human centromere and consisting of non-alpha-satellite DNA. Nat Genet 1997; 16: 144-153.

13 Depinet TW, Zackowski JL, Earnshaw WC et al: Characterization of neo-centromeres in marker chromosomes lacking detectable alpha-satellite DNA. Hum Mol Genet 1997; 6: 1195 -1204.

14 Rowe AG, Abrams L, Qu Y, Chen E, Cotter PD: Tetrasomy 15q25qter: Cytogenetic and molecular characterization of an analphoid supernumerary marker chromosome. Am J Med Genet 2000; 93: 393 - 398 .

15 Sjöstedt AW, Alatalo M, Wahlström, Köbeln UV, Olegard R: Replicaton error, a new hypothesis to explain the origin of a supernumerary marker chromosome in a mentally retarded boy. Hereditas 1989; 11: 115-123.

16 Dutly F, Balmer D, Baumer A, Binkert F, Schinzel A: Isochromosomes $12 \mathrm{p}$ and 9p: parental origin and possible mechanisms of formation. Eur J Hum Genet 1998; 6: 140-144

17 Eggermann T, Schubert R, Engels $\mathrm{H}$ et al: Formation of supernumerary euchromatic short arm isochromosomes: parent and cell stage of origin in new cases and review of the literature. Ann Génét 1999; 42: 75 -80.

18 Généthon, 2000: http://www.genethon.fr 